\title{
Hot coronal loops associated with umbral brightenings ${ }^{\star}$
}

\author{
C. E. Alissandrakis and S. Patsourakos
}

\author{
Department of Physics, University of Ioannina, 45110 Ioannina, Greece \\ e-mail: [calissan; spatsour]@cc.uoi.gr
}

Received 26 April 2013 / Accepted 21 June 2013

\begin{abstract}
Aims. We aim to investigate the association of umbral brightenings with coronal structures.

Methods. We analyzed AIA/SDO high-cadence images in all bands, HMI/SDO data, soft X-ray images from SXI/GOES-15, and $\mathrm{H} \alpha$ images from the GONG network.

Results. We detected umbral brightenings that were visible in all AIA bands as well as in $\mathrm{H} \alpha$. Moreover, we identified hot coronal loops that connected the brightenings with nearby regions of opposite magnetic polarity. These loops were initially visible in the $94 \AA$ band, subsequently in the $335 \AA$ band, and in one case in the $211 \AA$ band. A differential emission measure analysis revealed plasma with an average temperature of about $6.5 \times 10^{6} \mathrm{~K}$. This behavior suggests cooling of impulsively heated loops.
\end{abstract}

Key words. sunspots - Sun: corona - Sun: magnetic topology

\section{Introduction}

Sunspots are the site of many dynamic phenomena, such as umbral oscillations, running umbral/penumbral waves, and umbral flashes (see review by Solanki 2003). Beckers \& Tallant (1969) used the term umbral flashes to describe small (average diameter of $2200 \mathrm{~km})$, short-lived $(<120 \mathrm{~s})$ bright structures that they observed in the $\mathrm{K}$ line. They noticed a tendency of these flashes to repeat every $145 \mathrm{~s}$, which led many subsequent authors to identify them with the umbral oscillations discovered later by Bhatnagar \& Tanaka (1972), Beckers \& Schultz (1972) and Giovanelli (1972); however, the time profiles of umbral flashes presented by Beckers \& Tallant (1969) did not have a sinusoidal form, but showed a fast rise followed by a slower decay with a characteristic time of $\sim 50 \mathrm{~s}$.

Umbral flashes are difficult to observe in other chromospheric lines in the visible part of the spectrum. One case in $\mathrm{H} \alpha$ was reported by Alissandrakis et al. (1992). These authors were also the first to report on waves propagating from inside the umbra outward to become penumbral waves (Zirin \& Stein 1972; Giovanelli 1972). The propagating nature of umbral waves was subsequently verified by Alissandrakis et al. (1998) and Tsiropoula et al. (2000).

Another type of umbral brightenings, termed umbral flares, was reported by Tang (1978). They appear in $\mathrm{H} \alpha$ as bright patches confined inside the umbra, with the other footpoint located in the nearby plage; they last for $25-45 \mathrm{~min}$ and are sometimes accompanied by type III bursts.

An important aspect of sunspot-associated dynamic phenomena is their extension in the upper layers of the solar atmosphere. Indeed, Gelfreikh et al. (1999) detected sunspot oscillations at $1.7 \mathrm{~cm}$ with the Nobeyama radioheliograph, while Nindos et al. (2002) resolved them spatially using the VLA. The availability of continuous high-cadence, high-resolution observations of the entire sun from instruments onboard the

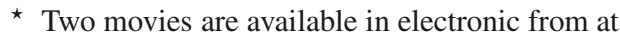
http: //www . aanda.org
Solar Dynamics Observatory (SDO) gives an excellent opportunity for detailed studies of sunspot-associated phenomena in the transition region and the corona (e.g. Reznikova et al. 2012).

In this article we used AIA/SDO data to investigate the extension of umbral brightenings into the transition region and the low corona. We describe the observations in Sect. 2 and discuss the results in Sect. 3 .

\section{Observations and results}

We examined SDO/AIA images of a number of isolated, welldeveloped symmetric sunspots with the help of the Helioviewer site for the occurrence of brightenings above their umbra. We found several cases, best visible in the $1600 \AA$ band, and selected two for more detailed study (Table 1); for this study we used cutouts of the original high-cadence images.

\subsection{Event of January 19, 2013}

A well-observed case is the event of January 19, 2013. Figure 1 shows a set of images in selected AIA wavelength bands together with HMI images of continuum intensity, longitudinal magnetic field, and line-of-sight velocity, as well as an $\mathrm{H} \alpha$ image from the GONG network, during the peak of the brightening. The bottom row shows images from which the intensity before the event was subtracted for better visibility of the brightening.

The brightening, located $3^{\prime \prime} \mathrm{N}$ of the spot center, is visible in all AIA bands; it is barely visible in the lower-resolution $\mathrm{H} \alpha$ difference image and there is no trace of it in the continuum, $B_{\ell}$ and $v_{\ell}$ images, not even in the image of the line depth of the FeI $6173 \AA$ line used by HMI (not shown here). Thus the lower extent of the brightening is somewhere between the formation height of the core of the FeI line $(\sim 302 \mathrm{~km}$, see Norton et al. 2006) and of the $1700 \AA$ band ( $360 \mathrm{~km}$, see Fossum \& Carlsson 2005). We found no indication of associated type III burst activity in the CALLISTO and WIND/WAVES data bases. The peak intensity of the brightening relative to the one before the event 
AIA, HMI \& H-alpha Images; Jan $192013115 " \times$ 90"

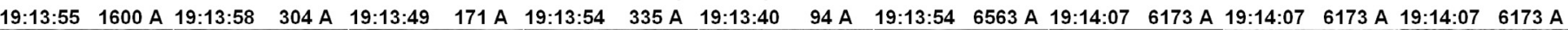

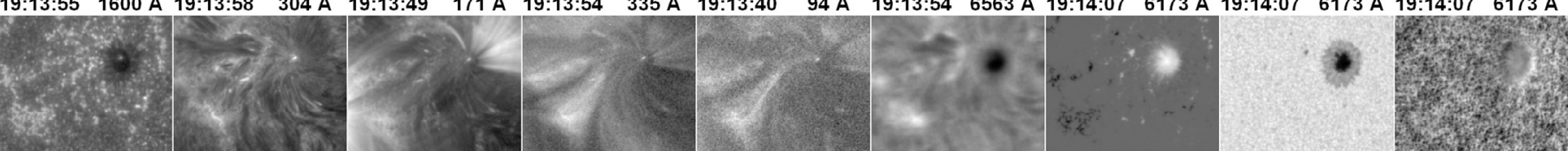

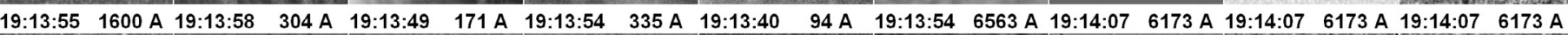

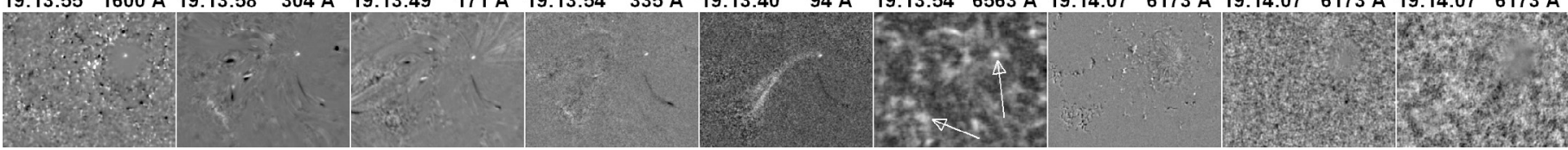

Fig. 1. Umbral brightening of January 19, 2013 in selected AIA bands and H $\alpha$, together with HMI images of the longitudinal magnetic field, continuum intensity, and line-of-sight velocity. The bottom row shows difference images. The arrows in the H $\alpha$ difference image point to the umbral brightening and the associated plage footpoint. The images are orientated in the solar E-W/N-S direction. A time sequence of images is shown in movie 1 in the electronic version of the journal. Each frame of the movie shows images in the 1600 and $304 \AA$ A bands (top row) as well as in the 94 and $335 \AA$ AIA bands (bottom row); the first frame shows direct images in all four bands, the others show difference images in all bands except $1600 \AA$.

Table 1. List of observations.

\begin{tabular}{lccc}
\hline \hline Date & Region & Location & Peak UT \\
\hline 2013 Jan. 19 & 1658 & W15.7 S12.0 & $19: 14$ \\
2012 Sep. 30 & 1579 & E01.8 S10.0 & $07: 53$ \\
\hline
\end{tabular}

Table 2. Peak intensity of brightenings relative to the local background.

\begin{tabular}{lccc}
\hline \hline Band & $\begin{array}{c}2013 \\
\text { Jan. 19 }\end{array}$ & $\begin{array}{c}2012 \\
\text { Sep. 30, E }\end{array}$ & $\begin{array}{c}2012 \\
\text { Sep. 30, W }\end{array}$ \\
\hline 1700 & 1.86 & - & 1.53 \\
1600 & 4.70 & 1.73 & 3.25 \\
304 & 3.74 & 3.00 & 3.08 \\
171 & 3.12 & 1.25 & 2.94 \\
211 & 2.66 & 1.90 & 1.48 \\
193 & 2.53 & 1.62 & 2.30 \\
131 & 3.26 & 2.50 & 3.08 \\
335 & 2.81 & 1.78 & 2.25 \\
94 & 3.00 & 2.26 & 1.80 \\
\hline
\end{tabular}

is given in Table 2; the brightening is strongest in the $1600 \AA$ band where its intensity is higher than that of the quiet Sun and similar to the average intensity of plage regions; the brightening is weakest in the $1700 \AA$ band.

At the time of its peak, the brightening had a rather simple structure (Fig. 2), with small differences among the AIA spectral bands. It was roughly elliptical in shape, with a FWHM of 2.4" by $1.4^{\prime \prime}$ in the $171 \AA$ image, with an arch-like extension NE; the structure was more compact in the 304, 335, and $94 \AA$ images, with the peak displaced slightly to the NW and some indication of a second peak.

The most interesting aspect of the brightening is that in the $94 \AA$ band image, with a characteristic temperature of $\log T \sim$ 6.8 (Lemen et al. 2012), it is clearly associated with a coronal loop (Fig. 1), best seen in the subtracted image. The loop ends in a region of opposite polarity at a projected distance of 53", which is bright in $\mathrm{H} \alpha$, and in the $304 \AA$ and some other AIA bands. This opposite footpoint is more extended than the brightening, and the cross-section of the loop along its length varies accordingly. This is consistent with the expected variation of the magnetic field strength from the sunspot to the plage.

The loop developed together with the brightening and was not detected in any other spectral band up to the maximum of the brightening. After that, the loop became progressively visible in
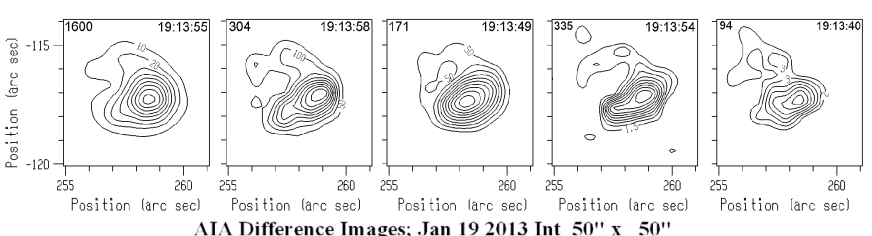

AIA Difference Images; Jan 192013 Int $50^{\prime \prime}$ x $50^{\prime \prime}$
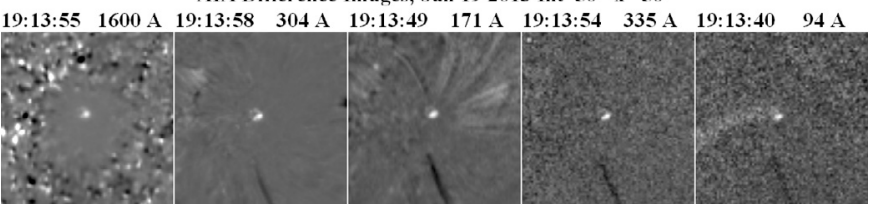

Fig. 2. Contour plots and difference images of the brightening at the time of its maximum.

the $335 \AA$ band (characteristic temperature of $\log T \sim 6.4$ ), while it faded away in the $94 \AA$ band (Fig. 3). Note that the loop persisted for several minutes after the brightening had faded away. Also note that the overall geometry of the loop and its footpoints is very well depicted in the images of the rms variation of the intensity (last row in Fig. 3).

No observations from Hinode were available at the time of the event. Soft X-ray images from SXI on GOES-15 show the loop in all channels, starting around 09:10 UT with a peak near the peak of the umbral brightening.

Figure 4 shows the time profiles of the brightening of two portions of the loop and of its southern footpoint. The time profiles of the brightening and the $\mathrm{S}$ footpoint during the decay phase in 94 and $335 \AA$ are probably contaminated by the loop emission. The brightening had two main peaks, a fast rise of a few minutes and a much longer decay. The duration of the main phase was shortest in the $1600 \AA$ band ( $\sim 10 \mathrm{~min})$. The $\mathrm{S}$ footpoint appeared almost simultaneously with the brightening at $304 \AA$, with a delay of $\sim 1 \mathrm{~min}$ in the rising phase and $\sim 3 \mathrm{~min}$ in the peak.

In the $94 \AA$ band the $\mathrm{S}$ and the $\mathrm{N}$ portions of the loop showed practically identical light curves, both delayed by $\sim 6$ min with respect to the peak of the brightening. In $335 \AA$ the $S$ footpoint appeared first, $\sim 12$ min after the peak of the brightening, followed by the $\mathrm{S}$ part of the loop after $\sim 3 \mathrm{~min}$ and the $\mathrm{N}$ part of the loop $\sim 7$ min later. Compared with the $94 \AA$ band, the N part of the loop in $335 \AA$ was delayed by $\sim 18 \mathrm{~min}$ and the $\mathrm{S}$ part by $\sim 12 \mathrm{~min}$. 
C. E. Alissandrakis and S. Patsourakos: Hot coronal loops associated with umbral brightenings

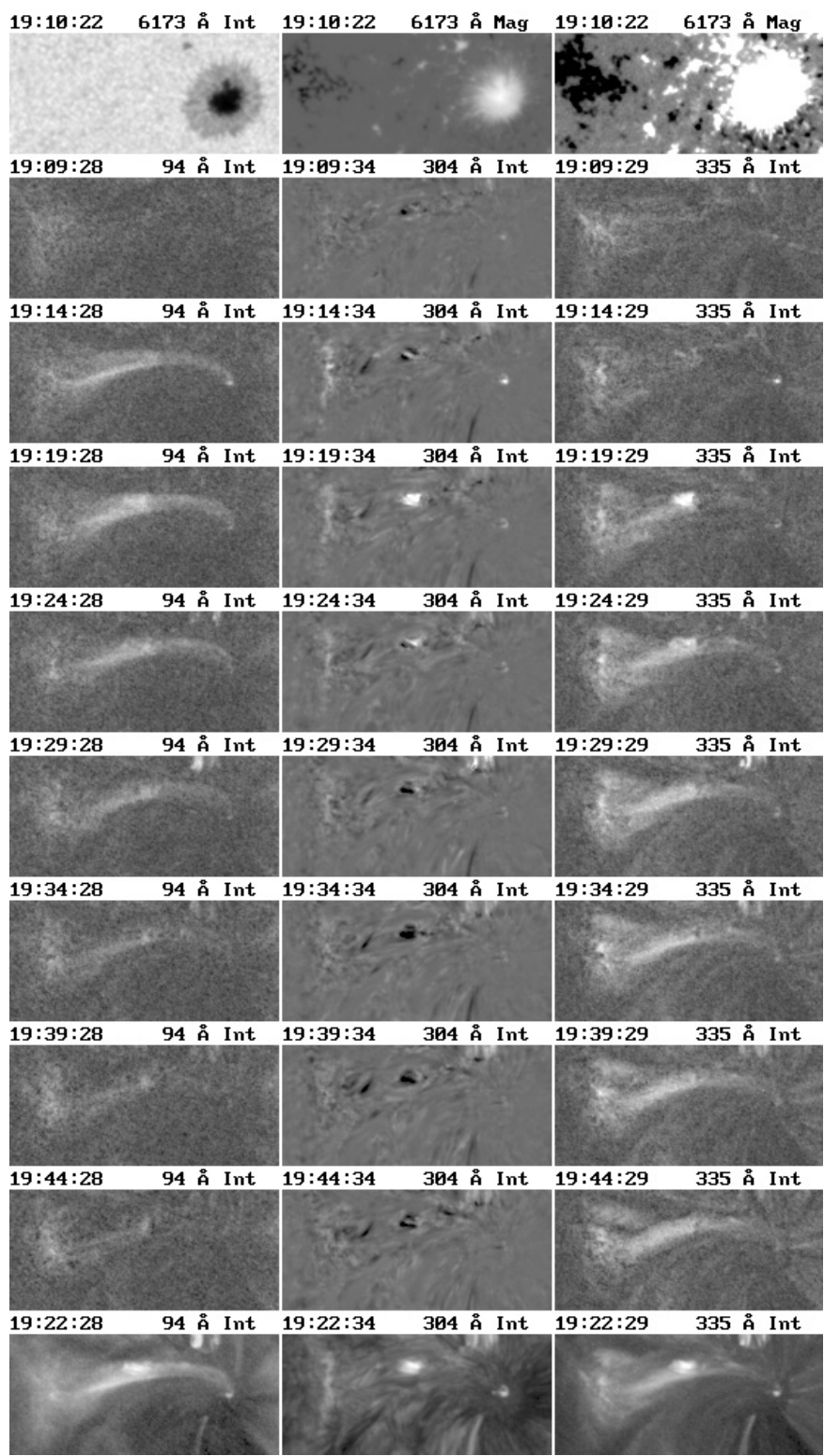

Fig. 3. Time sequence of difference images in the AIA 94, 304, and $335 \AA$ bands over a field of view of $100^{\prime \prime}$ by $45^{\prime \prime}$. The last row shows images of the intensity rms during the sequence (19:00 to 19:45 UT), while the first row shows HMI images of continuum intensity and longitudinal magnetic field, saturated at $\pm 100 \mathrm{G}$ in the right column. The 335 and $94 \AA$ images are one minute averages to reduce noise. The small bright structure near the middle of the loop is an unrelated phenomenon. The images have been rotated by $39^{\circ}$ with respect to the solar E-W direction.

To quantify the temperature structure of the loop we performed a differential emission measure (DEM) analysis based on the coronal AIA channels, using the method described in Plowman et al. (2013). Our results are presented in Fig. 5, which shows DEM images of the region for temperatures below $10^{6} \mathrm{~K}$ and at $10^{7} \mathrm{~K}$; in the first image the loop is invisible, while in the other it stands out clearly above the surrounding plasma. In the DEM images as a function of position and temperature shown in the bottom row of the figure, the bulk of the background plasma is in the temperature range $5.75<\log T<6.4$. In the lower left panel, where the DEM is displayed along a line crossing the loop at its middle, the loop appears at $x \sim 225$ as an excursion of
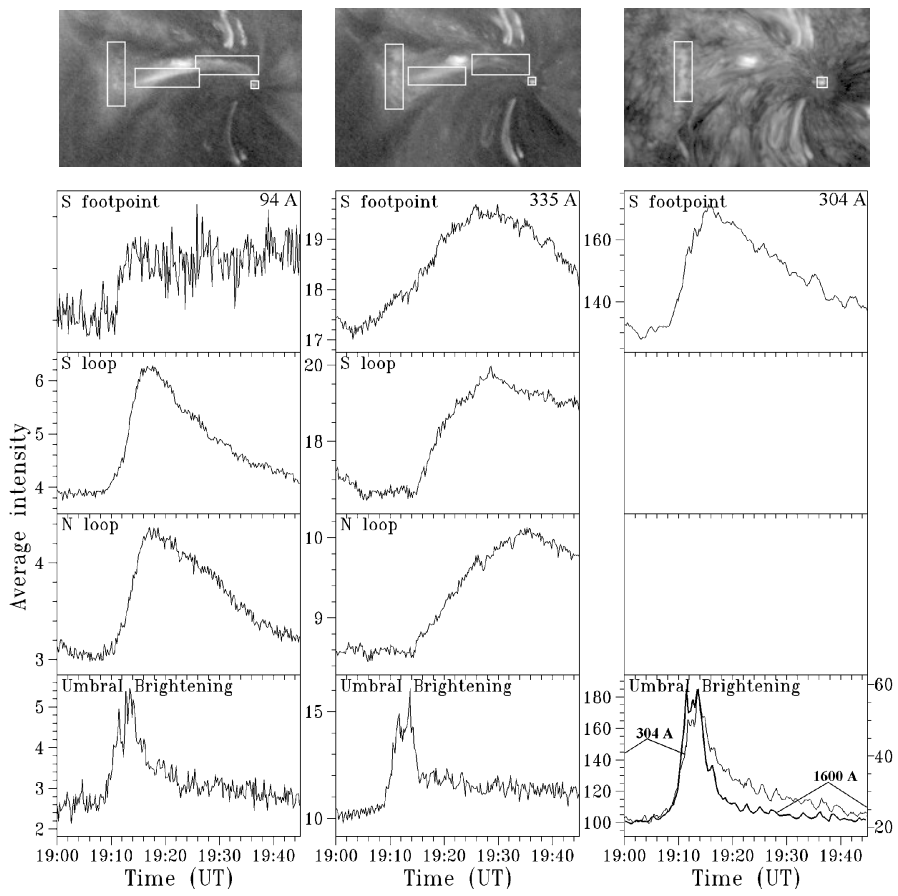

Fig. 4. Time profiles of the brightening, the southern footpoint and two portions of the loop. The boxes in the top row images show the corresponding regions in rms images (from left to right: $\mathrm{S}$ footpoint, $\mathrm{S}$ loop, $\mathrm{N}$ loop, umbral brightening).
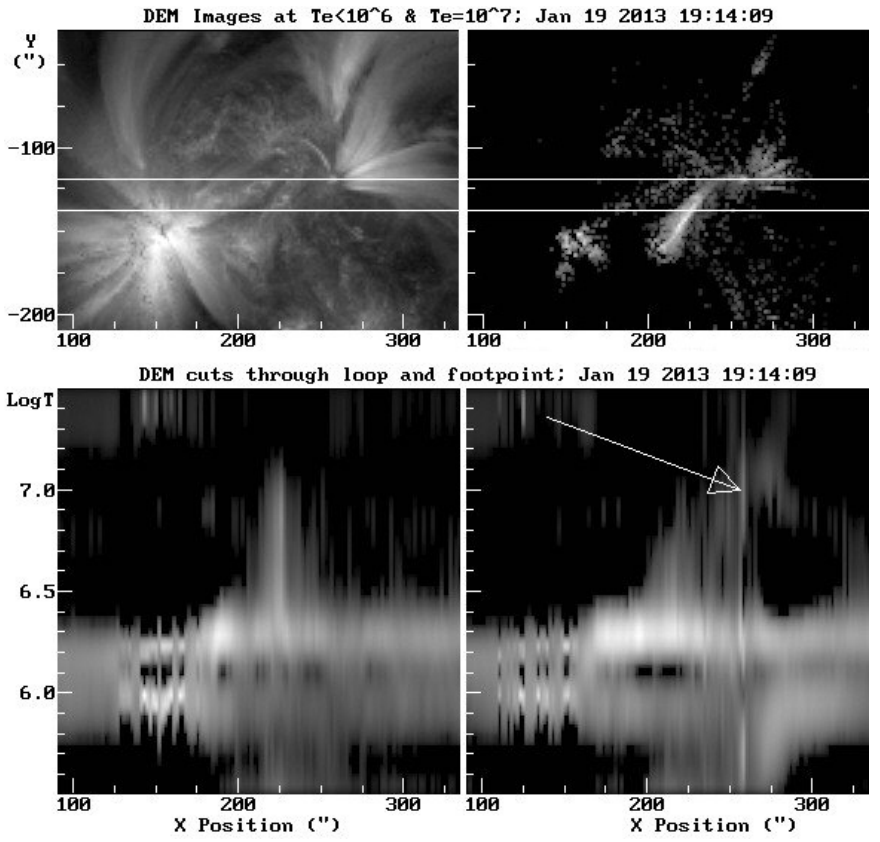

Fig. 5. Differential emission measure maps for $T<10^{6} \mathrm{~K}$ and $T=$ $10^{7} \mathrm{~K}($ top $)$. The bottom row shows the DEM as a function of position and temperature along the lines marked in the images at the top. The arrow marks the position of the brightening.

the DEM to high temperatures; the DEM peaks at $\log T \sim 6.82$ $\left(T \sim 6.5 \times 10^{6} \mathrm{~K}\right)$. The lower right panel of Fig. 5 shows the DEM along a line crossing the umbral brightening at $x=258$; here the DEM also extends to high temperatures, with a maximum at $\log T \sim 6.96$. 


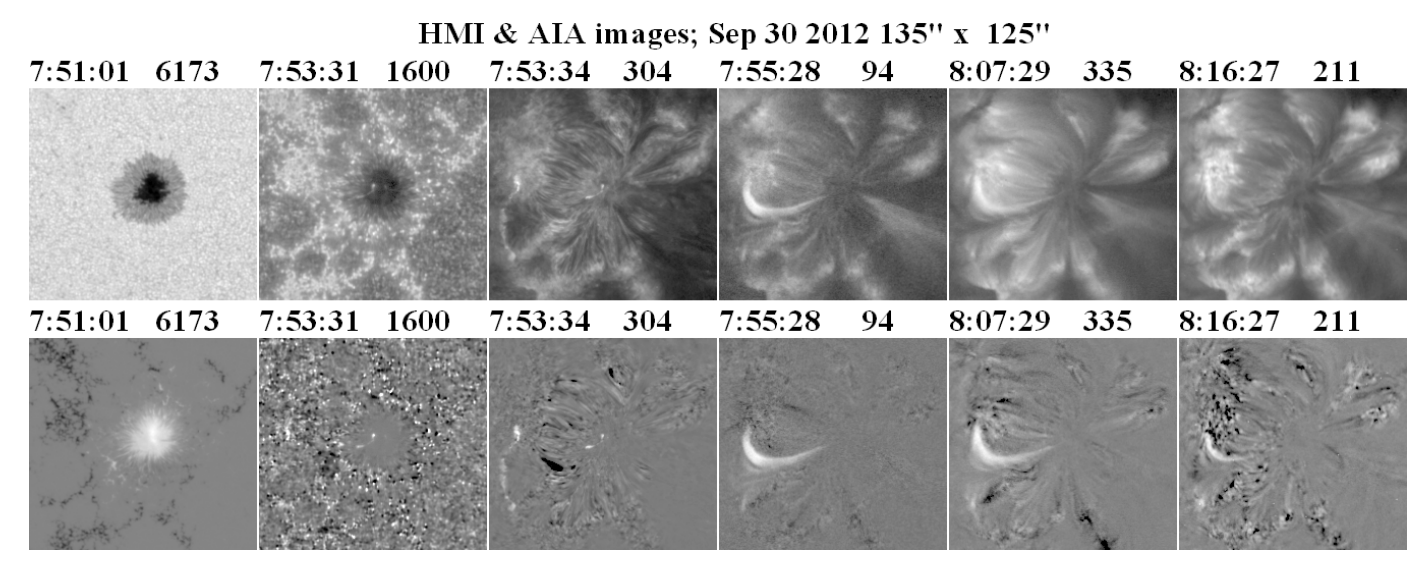

Fig. 6. Umbral brightening of September 30, 2012 in selected AIA bands. 1600 and $304 \AA$ images are at the peak of the brightening, 94, 335, and $211 \AA$ images are at the peak of the associated coronal loop. 94 and $335 \AA$ images are 1-min averages to reduce noise. The bottom row shows difference images. The first column shows HMI images of continuum intensity and longitudinal magnetic field. The time evolution of this loop and its footpoins is shown in movie 2 of the electronic version of the journal. Each frame of the movie shows difference images in the 1600 and $304 \AA$ bands (top row) as well as in the 94 and $335 \AA$ AIA bands (bottom row).

\subsection{Event of September 30, 2012}

A similar event was observed on September 30, 2012 in active region 1759 (Fig. 6). This brightening consisted of two patches, $10^{\prime \prime}$ apart, connected by a faint emission bridge that gave them a sickle-like appearance. The E patch started near the umbrapenumbra boundary and extended to the middle of the penumbra, the $\mathrm{W}$ patch was entirely within the umbra, $\sim 5^{\prime \prime}$ away from its center; their sizes were roughly 4 by $1.5^{\prime \prime}$ and 5 by $2^{\prime \prime}$ respectively, but both contained up to four smaller components, down to $1^{\prime \prime}$ size. A very weak type III burst was discernible during the rise phase of the W patch, at 07:51:09 UT in the San Vito dynamic spectra below $60 \mathrm{MHz}$ and in the WIND/WAVES RAD2 spectra (07:51 UT at 13.8 MHz and 07:52 UT at $2 \mathrm{MHz}$ ); lacking imaging data, we cannot associate them with certainty with the event.

The coronal loop started near the umbra-penumbra boundary, at the west end of the E patch and extended over $44^{\prime \prime}$ to the adjoining opposite polarity plage. The plage footpoint was visible not only in the $304 \AA$ band, but also in the $1600 \AA$ band (Fig. 6); it was also visible in the GONG difference $\mathrm{H} \alpha$ image, where the brightening itself was not. The time evolution of this loop is similar to that of the event of January 19, 2013: it was best visible in the $94 \AA$ band, $\sim 2$ min after the maximum of the umbral brightening and in the $335 \AA$ band $\sim 12$ min later. Here we also had emission in the $211 \AA$ band (characteristic temperature of $\log T \sim 6.3$ ), which peaked $\sim 23$ min after the brightening. Soft X-ray images from GOES SXI showed a picture similar to that of the January 192013 event: the hot loop was visible in all SXI channels and appeared at about the same time as the $94 \AA$ loop.

The light curve of the brightening itself had a fluctuating character, with the bulk of the emission lasting for $\sim 8 \mathrm{~min}$ in the $1600 \AA$ band. The peak intensities of the $\mathrm{E}$ and $\mathrm{W}$ components of the brightening relative to the local background are given in Table 2.

The results of our DEM analysis are shown in Fig. 7 at three instances during the evolution of the event. The background plasma now is near $\log T \sim 6.3$ and the cuts shown in the bottom row of the Figure cross the loop at two points, at $x=-80$ and $x=-45$; the DEM on the loop has a maximum at $\log T \sim 6.86$. Note also the gradual cooling of the loop between 07:53 UT and 08:15 UT.
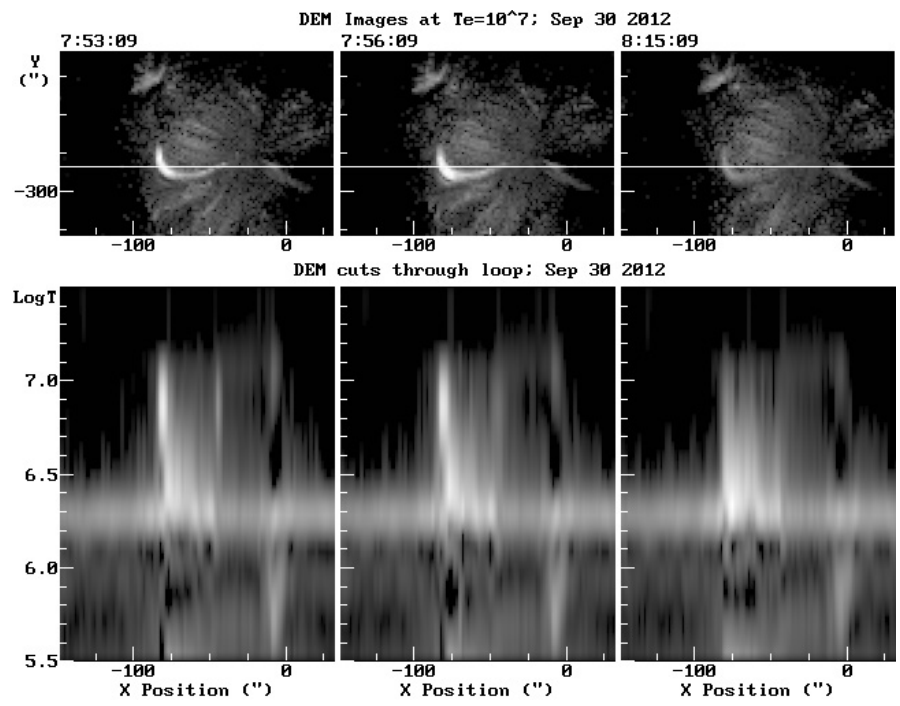

Fig. 7. Differential emission measure maps for $T=10^{7} \mathrm{~K}$ at three instances during the event of September 30, 2012 (top). The bottom row shows the DEM as a function of position and temperature along the line marked in the images at the top.

\section{Summary and discussion}

Our analysis of short-lived umbral brightenings from SDO/AIA data showed two cases where the brightenings were not confined to the low atmospheric layers. Not only were they visible in all AIA channels, which implies a very extended temperature range, but they were also connected by hot coronal loops to opposite-polarity footpoints located $\sim 50^{\prime \prime}$ away. The association of these brightenings with hot coronal loops proves beyond any doubt that their origin is magnetic. In this respect, they are similar to the umbral flares reported by Tang (1978), but they are more compact and of shorter duration. The brightenings were strongest in the $1600 \AA$ AIA band, where their intensity exceeded that of the quiet Sun and reached the average level of the plage. They did not appear in the HMI images, and we estimate their lowest extent to a height between $302 \mathrm{~km}$ and $360 \mathrm{~km}$.

We note briefly that the association with hot coronal loops is not a general property of umbral brightenings. We have found several cases that did not exhibit such an association (see, e.g. the 
bright point at $x=-26, y=-274$ at 07:42 UT in movie 2); these were shorter $(\sim 1 \mathrm{~min})$ and probably similar to the umbral flashes of Beckers \& Tallant (1969). We are currently preparing a report on them.

The associated hot coronal loops appeared first in the $94 \AA$ AIA band, where they reached maximum 2 to 6 min after the brightening maximum. Subsequently, they became visible in the $335 \AA$ band and, in the case of the September 302012 event, in the $211 \AA$ band. In the GOES-15 SXI soft X-ray images the loop was visible in all bands with a peak near that of the $94 \AA$ loop. This behavior is suggestive of gradual cooling of hot plasma.

The high temperature of the loops is confirmed not only by their appearance in the hot $94 \AA$ channel and in the SXI images, but also by our DEM analysis, which showed that the average temperature of the loop plasma was $\sim 6.5 \times 10^{6} \mathrm{~K}$. Thus the events presented here are clear examples of plasma cooling from high temperatures of several MK to $\approx 2.5 \mathrm{MK}$. The fact that the hot loops are rooted in sunspot umbrae is a key advantage of our data, since this allows direct observation of their footpoints without obscuration from low-lying structures. Obscuration is a limiting factor in observations of loops rooted in plages, where it is often hard to obtain a view of their footpoints and is difficult even to identify them. This is the case for several studies of hot evolving coronal loops in active regions using Hinode and AIA (e.g. Warren et al. 2011).

Cooling of individual coronal loops or even of large 2D fields in ARs has been observed in the past (e.g. Warren et al. 2003; López Fuentes et al. 2007; Ugarte-Urra et al. 2009; Viall \& Klimchuk 2011; Warren et al. 2011). There are two possibilities to explain the cooling. One is that we may have a quasi-steady hot coronal loop that begins to cool down once its (quasi-steady) heating is shut off. This possibility can be safely excluded, because quasi-steady multi-million degrees hot coronal loops are expected to have intense footpoint emissions (e.g. Patsourakos $\&$ Klimchuk 2008). However, our observations of the umbral loop footpoints show that they are only a factor $\approx 2-3$ more intense than the associated coronal sections. A second, more plausible scenario for the observed evolution is that the coronal-loop cooling follows its impulsive heating to multi-million $\mathrm{K}$. It is possible that the heating phase cannot be observed due to the low emission measure associated with it: the temperature increase is not initially tracked by the loop density, because it takes some time to fill a coronal loop with hot plasma via chromospheric evaporated material (e.g. Patsourakos \& Klimchuk 2006).

We point out here that high-frequency impulsive heating, with a repeat time between successive heating pulses shorter than the coronal cooling time, also leads to quasi-steady conditions. If this had been the case, one would expect re-heating of the observed loops. However, inspection of AIA movies spanning over 90 min after the appearance of the observed loops did not show strong evidence for this. The fact that the observed loops did not appear to cool down to the 211 channel and below may imply that they did not have enough emission to stand above the strong, highly-structured and dynamic active-region background observed in warmer emissions ( $\leq 2 \mathrm{MK})$. Our inferences above would be more firmly confirmed or rejected through detailed modeling of the observed loops, which is an important task for the future.

The relatively simple geometry of the hot loops and their footpoints as well as the possibility of following their temperature-density evolution through computating the DEM from AIA data makes these structures ideal for studying the heating and cooling of loops; we intend to do this in a future work.

Acknowledgements. The authors made extensive use of the data bases of SDO (AIA and HMI), GONG, GOES, CALLISTO, WIND/WAVES, the USAF RSTN network and the Helioviewer site (http://helioviewer.org); they are grateful to all those that worked for the development and operation of these instruments and for making the data available. The research of SP has been supported in part by the European Union (European Social Fund ESF) and in part by the Greek Operational Program "Education and Lifelong Learning" of the National Strategic Reference Framework (NSRF) - Research Funding Program: Thales "Hellenic National Network for Space Weather Research"-MIS 377274. S.P. also acknowledges support from an FP7 Marie Curie Grant (FP7-PEOPLE-2010$\mathrm{RG} / 268288$ ).

\section{References}

Alissandrakis, C. E., Georgakilas, A. A., \& Dialetis, D. 1992, Sol. Phys., 138, 93 Alissandrakis, C. E., Tsiropoula, G., \& Mein, P. 1998, ASP Conf. Ser., 155, 49 Beckers, J. M., \& Schultz, R. B. 1972, Sol. Phys., 27, 61

Beckers, J. M., \& Tallant, P. E. 1969, Sol. Phys., 7, 351

Bhatnagar, A., \& Tanaka, K. 1972, Sol. Phys., 24, 87

Fossum, A., \& Carlsson, M. 2005, ApJ, 625, 556

Gelfreikh, G. B., Grechnev, V., Kosugi, T., \& Shibasaki, K. 1999, Sol. Phys., 185,177

Giovanelli, R. G. 1972, Sol. Phys., 27, 71

Lemen, J. R., Title, A. M., Akin, D. J., et al. 2012, Sol. Phys., 275, 17

López Fuentes, M. C., Klimchuk, J. A., \& Mandrini, C. H. 2007, ApJ, 657, 1127

Nindos, A., Alissandrakis, C. E., Gelfreikh, G. B., Bogod, V. M., \& Gontikakis, C. 2002, A\&A, 386, 658

Norton, A. A., Graham, J. P., Ulrich, R. K., et al. 2006, Sol. Phys., 239, 69

Patsourakos, S., \& Klimchuk, J. A. 2006, ApJ, 647, 1452

Patsourakos, S., \& Klimchuk, J. A. 2008, ApJ, 689, 1406

Plowman, J., Kankelborg, C., \& Martens, P. 2013, ApJ, 771, 2

Reznikova, V. E., Shibasaki, K., Sych, R. A., \& Nakariakov, V. M. 2012, ApJ, 746,119

Solanki, S. K. 2003, A\&ARv, 11, 153

Tang, F. 1978, Sol. Phys., 60, 119

Tsiropoula, G., Alissandrakis, C. E., \& Mein, P. 2000, A\&A, 355, 375

Ugarte-Urra, I., Warren, H. P., \& Brooks, D. H. 2009, ApJ, 695, 642

Viall, N. M., \& Klimchuk, J. A. 2011, ApJ, 738, 24

Warren, H. P., Winebarger, A. R., \& Mariska, J. T. 2003, ApJ, 593, 1174

Warren, H. P., Brooks, D. H., \& Winebarger, A. R. 2011, ApJ, 734, 90

Zirin, H., \& Stein, A. 1972, ApJ, 178, L85 